\title{
Auditory temporal order and perceived fusion-nonfusion
}

\author{
GREGORY M. CORSO \\ Georgia Institute of Technology, Atlanta, Georgia 30332
}

\begin{abstract}
A pair of pure-tone sine waves, with the first tone presented randomly to either ear, was presented simultaneously or sequentially. The order of occurrence of the tones (temporal order) and the number of tones perceived (fusion-nonfusion) were judged. Three values of stimulus intensity $(40,55$, and $70 \mathrm{~dB}$ SPL) and 10 values of stimulus onset asynchrony (SOA; 0, 1, 2, and 4 to $28 \mathrm{msec}$ in 4 -msec steps) were varied. Of major importance in this investigation was the relationship between the judgmental tasks of temporal order and fusionnonfusion. Response time and accuracy were the dependent measures used to assess that relationship. The results of two groups of 10 subjects showed an increase in the percentage of correct nonfusion judgments with increases in stimulus intensity and SOA. For correct judgments of temporal order, no significant intensity or SOA effects were noted. Response time for the fusion-nonfusion task was significantly influenced by both stimulus intensity and SOA. For the response times associated with the temporal order task, intensity was significant. More important to this investigation was the observed Intensity by SOA by Task interaction for response time data. These results suggest a hybrid model, with different processes occurring within different stages of this model.
\end{abstract}

Temporal order and fusion-nonfusion information extracted from two stimuli is functionally related to the temporal interval between the onsets of the stimuli (Babkoff, 1975; Babkoff \& Sutton, 1966; Cheatham \& White, 1954; Corso, 1976, 1978; Hirsh, 1959; Hirsh \& Sherrick, 1961; Sternberg \& Knoll, 1973; Stroud, 1955; White, 1963; White \& Lichtenstein, 1963). Intervals of less than $10 \mathrm{msec}$ will result in the perception of one fused stimulus located somewhere between the two ears (Babkoff, 1975; Corso, 1978; Fraisse, 1963). The location depends on the intensity of the stimuli and the phase relation between the two stimuli (Harris, 1974). However, if that interval is greater than $10 \mathrm{msec}$, then two nonordered stimuli will be perceived (Babkoff, 1975; Corso, 1978; Fraisse, 1963). Perception of order begins to occur when this interval exceeds $20 \mathrm{msec}$ (Hirsh \& Sherrick, 1961; Corso, 1976).

The present study was designed to extend this work by investigating the relationship between the judgmental tasks of temporal order and fusion-nonfusion. Babkoff (1975), Hirsh and Sherrick (1961), and Sternberg and Knoll (1973) have implied that judgments of temporal order may require information from prior processing stages; that is, temporal order judgments are derived from at least a two-stage, but more probably a multistage, sequential process. Because of the greater SOA-threshold value for temporal order relative to the SOA-threshold value for fusionnonfusion, and on the assumption that response latency reflects the dependency of temporal order judgments on fusion-nonfusion judgments, two hypotheses were tested:

(1) If accurate judgments of temporal order require information about perceptions of nonfusion, then, as SOA increases, the accurate perception of order should remain invariant until increases in SOA result in the perception of two stimuli. Once two stimuli have been perceived, then increases in the accuracy of the order judgment should result. The hypothesized functions are presented in Figure 1A.

(2) The latencies for the temporal order task at each SOA value should be longer than those for the fusionnonfusion task. Moreover, as SOA increases, the latencies associated with both tasks should decrease. These hypothesized functions, presented in Figure 1B, reflect the assumption that subsequent stages of processing increase the latency of the judgments. Since it has been proposed that temporal order judgments require more processing stages than do fusion-nonfusion judgments (Babkoff, 1975), longer latencies for the temporal order judgments should be observed.

The present study also sought to determine the influence of stimulus intensity on the response latency and accuracy measures associated with the judgmental tasks of temporal order and fusion-nonfusion. Given the assumption of a multistage sequential process, the influence of stimulus intensity on the processes within each stage should be similar, given similar processes. However, while stimulus intensity has been found to be inversely related to the fusionnonfusion threshold (Fraisse, 1963), the influence of 

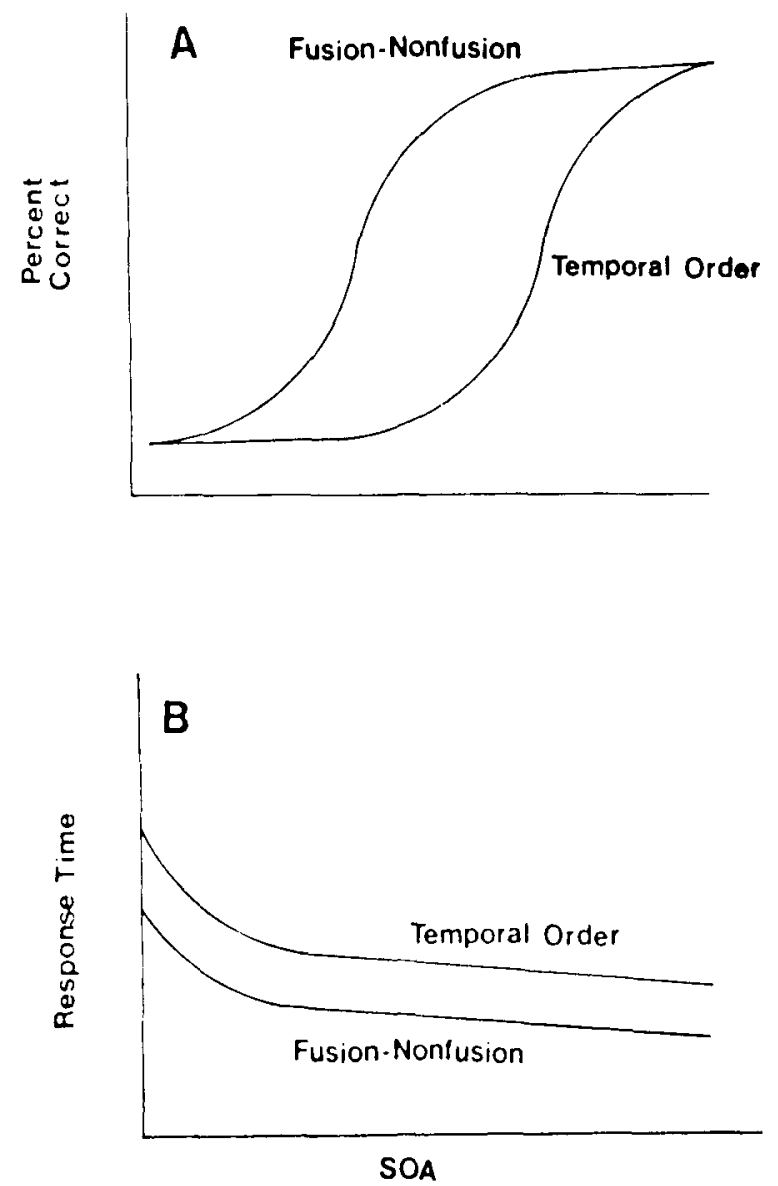

Figure 1. The hypothetical functions relating percent correct and response time for temporal order and nonfusion judgments as a function of SOA.

stimulus intensity on the temporal order threshold is conflicting (Corso, 1978; Rutschmann, 1973). Hirsh and Sherrick (1961) have shown that stimulus intensity does not alter the temporal order threshold; however, Rutschmann (1973) has shown that the temporal order threshold is inversely related to stimulus intensity.

Moreover, the manipulation of stimulus intensity may provide a means of incorporating the judgmental tasks of the present study into one of the reaction time models (Sternberg \& Knoll, 1973), provided that the task latencies are inversely related to stimulus intensity, as is simple reaction time (Teichner \& Krebs, 1972).

\section{METHOD}

\section{Subjects}

Six male and 14 female student volunteers from a course in introductory psychology served as subjects. Three males and 7 females were assigned randomly to one of the two task groups. Initially, the subjects were screened for normal hearing at the frequencies of $500,1,000,2,000,4,000$, and $8,000 \mathrm{~Hz}$, with a Tracor Clinical Audiometer (Model 115A), and were required to have a hearing level of at least $20 \mathrm{~dB}$. In addition, the subjects were required to be between the ages of 18 and 30 years, right-handed, and English-speaking. All subjects served in a single 2-h session.

\section{Apparatus}

A specially designed and constructed apparatus was used in this experiment. The apparatus generated a sine-wave signal of $1,000 \mathrm{~Hz}$. The duration, rise and fall times, and attenuation of the signal were controlled through a keyboard. The apparatus was constructed so that the signal could appear in both ears either simultaneously or offset by specific, user-controlled, SOA values, also input through the keyboard. Additionally, the apparatus contained two response clocks and two response indicators.

The auditory stimuli were presented to the subjects through a matched and calibrated set of Grason-Stadler TDH-49 earphones, fitted with MX-41/AR cushions.

A panel, containing a central "home key" and two response keys as well as a warning light, was used to designate the response. For the temporal order task, the two response keys designated the right ear and the left ear; for the fusion-nonfusion task, they designated fusion and nonfusion.

The subject's testing station was situated in a homemade sound-deadened chamber. All experimental apparatus, with the exception of the response panel, was situated in an adjacent room. Viewing of the subject was possible through a one-way mirror.

Design

The judgmental task (temporal order or fusion-nonfusion) was used as a between-subject variable, while stimulus onset asynchrony and stimulus intensity were within-subject variables. Stimulus onset asynchrony 10 values, and stimulus intensity assumed three values (see Stimulus section for details).

The 10 SOA values were combined factorially with the three intensity values so that each intensity value occurred with each SOA value. Furthermore, the initial stimulus of the stimulus pair was randomly presented to the right ear on one-half of the trials and to the left ear on the other half of the trials. The Intensity by SOA by Initial Ear of Stimulus Presentation combinations were presented randomly to each subject. Given the completely randomized design, 60 judgments were required from each subject. Additionally, there were five replications of the factorial design for each subject, providing a total of 360 trials per subject, or 7,200 judgments for the experiment.

\section{Stimuli}

The stimuli were two pure-tone sine waves with a frequency of $1,000 \mathrm{~Hz}$. They were presented either to both ears at the same time (diotic presentation) or to both ears at different times (dichotic presentation). For all presentations, the two stimuli were in phase.

Each stimulus within the stimulus pair was equal in intensity; however, the intensity between the pairs of stimuli assumed values of 40,55 , and $70 \mathrm{~dB}$ SPL (re .0002 microbar). Additionally, each stimulus assumed a constant duration of $20 \mathrm{msec}$, which included a linear 10-msec rise-and-fall time.

Stimulus onset asynchrony values were fixed at $0,1,2$, and 4 to $28 \mathrm{msec}$ in 4 -msec steps, providing a total of 10 SOA values.

The frequency of the stimulus was calibrated by means of a Hewlett-Packard digital counter, Model 5302A. The intensity of the signal was calibrated with a Brüel and Kjaer precision sound level meter, Model 2203, and a Brüel and Kjaer artificial ear, Model 4152, fitted with a T4131 condenser microphone.

\section{Procedure}

After the initial screening session, each subject was required to perform a simple reaction time task to a $1,000-\mathrm{Hz}$ diotically presented auditory stimulus pair. A red warning light occurred 200 to $700 \mathrm{msec}$ (variable in $100-\mathrm{msec}$ steps) prior to the onset of the first stimulus of the stimulus pair. The variable interval was used to prevent response anticipation. The duration of the warning light was $100 \mathrm{msec}$. The intensity of the stimulus assumed 
values of 40,55 , and $70 \mathrm{~dB}$ SPL. For the first 75 trials, the subject responded to the onset of the stimulus by releasing, with the index finger of the right hand, the home key and then depressing either the right or left response key and returning to the home key; the home key was again depressed until the next stimulus was presented. On the remaining 75 trials, the alternate response key was depressed. Upon completion of the simple reaction time task, a 5-min rest period was given.

In the final portion of the experiment, each subject was required to perform one of the following tasks:

Fusion task. Following the occurrence of the warning signal and variable interval, the stimulus pair was presented. The subject was required to report the perception of one or two stimuli. Fusion was defined as the report of one stimulus, and nonfusion, as the report of two stimuli. The subject responded by releasing, with the index finger of the right hand, the home key and depressing one of the two response keys to designate the number of stimuli perceived. After depressing the response key, the subject returned to the home key and depressed that key until the next stimulus was presented.

Temporal order task. Following the warning signal and variable warning interval, the stimulus pair was presented. The subject was required to report the order of occurrence of the two stimuli by judging whether the stimulus occurred first in the right ear or first in the left ear. The response for this task was performed in the same manner as in the fusion task, but the two keys designated the right and left ears.

\section{RESULTS}

The mean reaction time values obtained from the simple reaction time task were subjected to an analysis of variance for repeated measures. The purpose of this analysis was to establish that no reaction time differences existed between groups and response keys. No significant differences or interactions for those two variables were observed. However, the main effect of stimulus intensity was significant $[F(2,36)=$ $4.09, \mathrm{p}<.05]$. A least-significant-difference test of the response times associated with the three intensity values showed that the $40-70-\mathrm{dB}$ and $40-55-\mathrm{dB}$ differences were significant. There was an inverse relationship between stimulus intensity and reaction time, such that, as intensity increased, simple reaction time decreased.

\section{Accuracy of Temporal Order and Fusion Judgments}

The number of correct responses was summed separately for each subject across the 12 ( 2 ear by 6 replications) Intensity by SOA Trials for the temporal order and fusion tasks. A percent correct score was then calculated. The percentages for each subject were then subjected to a split-plot analysis of variance. For those trials in which the SOA value was $0 \mathrm{msec}$, the response was excluded from the analysis.

The results of this analysis showed a significant main effect for intensity $[F(2,36)=10.76, p<.01]$ and SOA $[\mathrm{F}(8,144)=65.10, \mathrm{p}<.01]$. Additionally, significant interactions were observed for Intensity by Task $[\mathrm{F}(2,36)=6.83, \mathrm{p}<.01]$, SOA by Task $[\mathrm{F}(8,144)$ $=52.88, \mathrm{p}<.01]$, SOA by Intensity $[\mathrm{F}(16,288)=2.03$, $\mathrm{p}<.01]$, and Intensity by SOA by Task $[\mathrm{F}(16,288)$ $=3.10, \mathrm{p}<.01]$.

The three-way interaction is presented in Figure 2 for the fusion and temporal order tasks. The function for the fusion task, presented in Figure 2A, shows the usual increase in percent correct as SOA increases. Furthermore, the percent correct for the fusion task is significantly influenced by stimulus intensity when the SOA values are greater than $4 \mathrm{msec}$. The percent correct judgments for the temporal order task, presented in Figure 2B, show a rather pronounced and significant $(\mathrm{p}<.01)$ influence of stimulus intensity at the 2-msec SOA value. Other than the increase in the percent of correct temporal order judgments from 1 to $2 \mathrm{msec}$, and the convergence of the function at $4 \mathrm{msec}$, the percentage of correct judgments for the temporal order task are constant for intensity and SOA, at least up to $28 \mathrm{msec}$.

\section{Response Times for Accurate Judgments}

It was hypothesized that if judgments of fusion and nonfusion occurred prior to judgments of temporal order, then the response time functions associated with each task should be parallel, with the fusion-nonfusion function resulting in a faster response time for all SOA values.

For each subject, the median correct response time was calculated for the 12 ( 2 ear by 6 replications) trials for each level of intensity, SOA, and task. In order to accommodate the data to the analysis of variance, a correct response time was defined as that response time associated with a response that occurred greater than $50 \%$ of the time over the 12 ( 2 ears by 6 replications) trials. The medians were

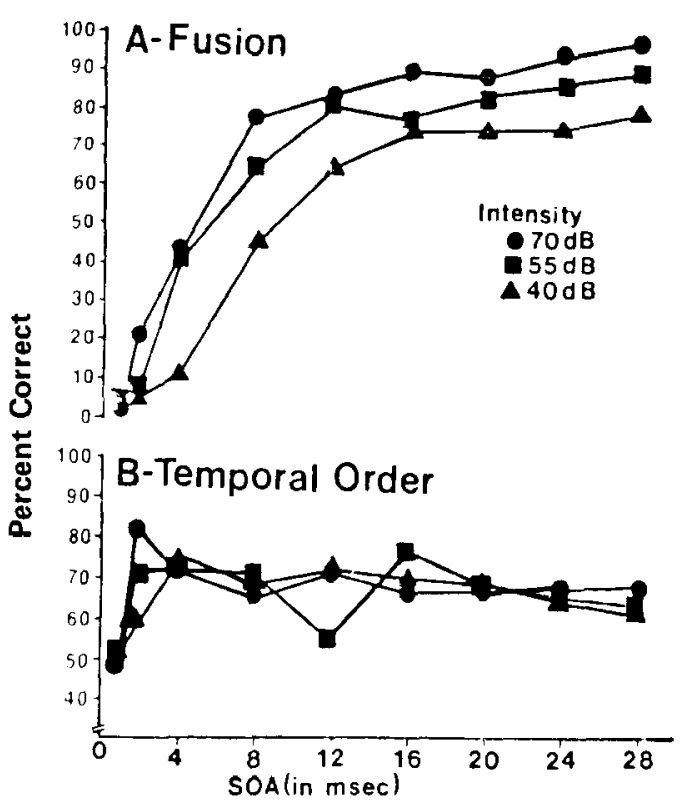

Figure 2. The observed Intensity by SOA by Task interaction for percent correct. 
then transformed to a log-base-10 score. The transformed medians were subjected to a split-plot analysis of variance with 2 levels of task, 3 levels of intensity, and 9 levels of SOA (the 0 -msec SOA value was not used in the analysis). The results of the analysis showed that the main effects of intensity $[F(2,36)=29.62, p<.01]$ and $\operatorname{SOA}[F(8,144)=2.65$, $\mathrm{p}<.01]$ were significant. Additionally, the SOA by Task $[F(8,144)=3.056, p<.01]$, the Intensity by SOA $[F(16,288)=2.19, \mathrm{p}<.01]$, and the Intensity by SOA by Task $[F(16,288)=3.75, p<.01]$ interactions were significant.

The three-way interaction, presented in Figure 3, was evaluated to assess the significant differences between the two tasks for the same intensity level at each SOA value. The evaluation showed that at

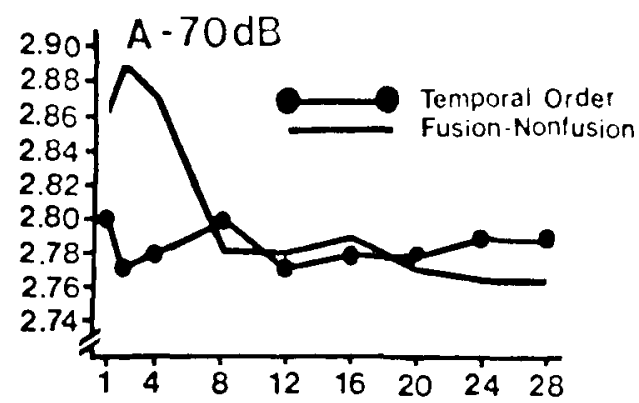

ठ
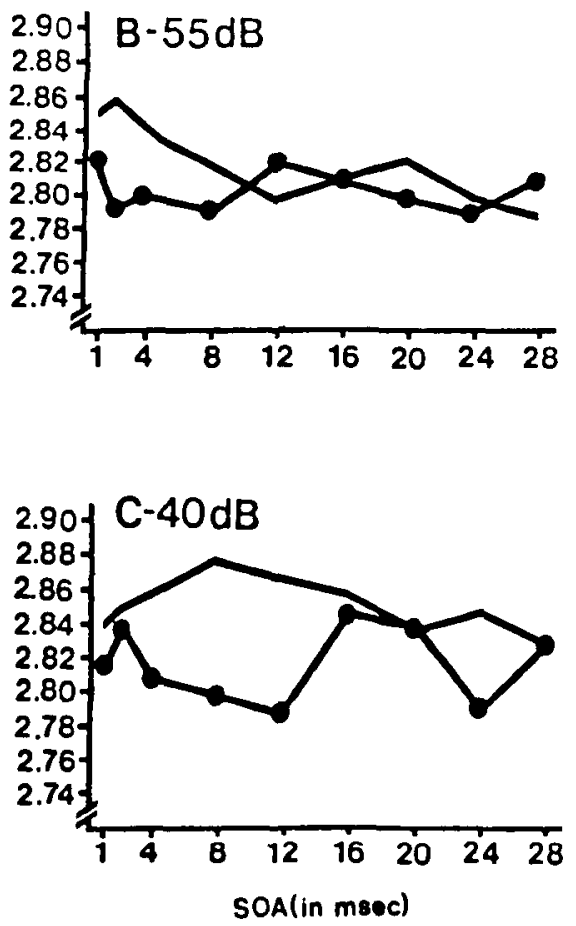

Figure 3. The observed Intensity by SOA by Task interaction for response time as a function of SOA, with task as the parameter. the 70-dB intensity level, presented in Figure 3A, the two tasks were significantly different for the SOA values of 1,2 , and $4 \mathrm{msec}$, with the temporal order task requiring less time. No significant differences between the two tasks were observed for SOA values from 8 to $20 \mathrm{msec}$, inclusive. For SOA values greater than $20 \mathrm{msec}$, the temporal order task required significantly more time than the fusion task.

For the 55-dB intensity value, presented in Figure $3 \mathrm{~B}$, the $1-\mathrm{msec} \mathrm{SOA}$ value resulted in no significant difference between the two tasks. With SOA values of 2 and $4 \mathrm{msec}$, the fusion task required more time than the temporal order task. For SOA values from 8 to $28 \mathrm{msec}$, inclusive, no significant differences between the tasks were observed.

In a similar manner, for the $40-\mathrm{dB}$ intensity value, presented in Figure 3C, the SOA values of 1 and $2 \mathrm{msec}$ resulted in no significant differences between the tasks. With SOA values from $4 \mathrm{msec}$ to less than $16 \mathrm{msec}$, more time was required for the fusion task. Stimulus onset asynchrony values of $16-20 \mathrm{msec}$ resulted in no significant task differences. The $24-\mathrm{msec}$ SOA value resulted in the fusion task's requiring more time than the temporal order task. No significant difference was observed for the $28-\mathrm{msec}$ SOA value.

Separate analyses were performed on the two tasks to determine if intensity and SOA were differentially related to the response times associated with each task.

For the fusion task, intensity was significant $[\mathrm{F}(2,18)$ $=14.15, \mathrm{p}<.01]$, SOA was significant $[\mathrm{F}(8,72)=5.34$, $\mathrm{p}<.01]$, and the interaction between intensity and SOA was significant $[F(16,144)=5.63, p<.01]$. The function relating intensity and SOA to the response time for the fusion task is presented in Figure 4A. This figure shows that, for SOA values greater than $8 \mathrm{msec}$, stimulus intensity is inversely related to response time.

For the temporal order task, neither SOA nor the Intensity by SOA interaction had any effect on the response time. Intensity was significant $[F(2,18)=$ $21.39, \mathrm{p}<.01]$ and is presented with SOA on the abscissa in Figure 4B. This figure shows that intensity was effective at the 2-msec SOA value and at the $16-$ and $20-\mathrm{msec}$ values.

\section{DISCUSSION}

The results of this experiment partially support Hypothesis 1; that is, the perception of temporal order remained invariant, while the accuracy of nonfusion judgments increased. However, for very short onset delays, temporal order accuracy was superior to nonfusion accuracy. This result would seem to suggest that, for very short intervals, temporal order information is used in judgments of fusion-nonfusion. While it must be noted that the traditional threshold 


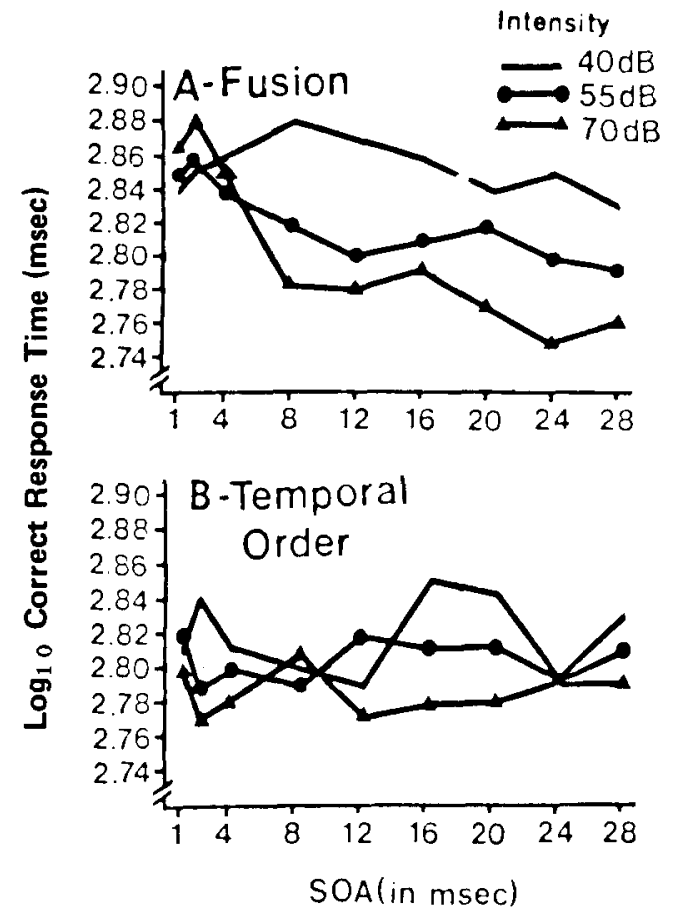

Figure 4. The observed Intensity by SOA by Task interaction for response time as a function of $\mathrm{SOA}$, with intensity as the parameter.

for temporal order is $75 \%$ correct (Hirsh \& Sherrick, 1961), even if the temporal order function were modified so that both the nonfusion and temporal order functions originated at the $50 \%$ and the $75 \%$ points, respectively, the predicted function still would not be realized. Moreover, it must be noted that the level of accuracy for the temporal order task obtained in this investigation does not agree with the data obtained from other temporal order investigations. Usually $75 \%$ correct occurs with an onset delay of from 15 to $30 \mathrm{msec}$. The lower accuracy level resulting from this investigation may have been the result of different stimulus intensities, naive subjects, or different stimulus durations.

Likewise, the response time data only partially support Hypothesis 2; that is, the response time function for the correct temporal order judgments was not greater than that for the fusion-nonfusion judgments for all onset delays. It must be noted, however, that the response time function for the fusion task is essentially composed of two distinct functions. One function is from a $1-\mathrm{msec}$ onset delay to the nonfusion threshold (a point that varies with intensity and that can be determined by locating the $50 \%$ point for a specific intensity in Figure 2) and is associated with a perception of fusion. The second function is from the threshold to $28 \mathrm{msec}$ and is associated with a perception of nonfusion. Therefore, three separate relations appear to have resulted from the response time data. Depending on the intensity of the stimuli, for very short onset delays, fusion judg- ments require more processing time than temporal order judgments. Since the two stimuli are fused during these intervals, the temporal order judgment, as defined in this task, may be more appropriately considered to be a lateralization judgment. Consequently, it would appear that lateralization judgments occur prior to, and do not require information about, fusion. In fact, given the response time data in addition to the accuracy data, lateralization judgments appear to be fundamental for decisions of fusion as opposed to the findings of Wallach, Newman, and Rosenzweig (1949), in which it was suggested that a perception of fusion was fundamental for a perception of lateralization. Moreover, the intersection of the functions relating the latency of the fusion judgment with the temporal order judgment corresponds with the interval of 8 to $16 \mathrm{msec}$ found by Babkoff (1975) for the break-up of the fused image and the perception of temporal order.

Additional increases in onset delay result in no response time differences for the two tasks. For these onset delays, temporal order judgments and nonfusion judgments require the same amount of processing time. It could be speculated that during this interval, since nonfusion judgments are in the range of $75 \%$ to $100 \%$ correct, the perception is one of either successiveness or simultaneity.

Lastly, as onset delay increases even more, it appears (from the $70-\mathrm{dB}$ condition) that temporal order judgments require more time than nonfusion judgments. This finding is consistent with the data provided by Babkoff (1975) in which temporal order perceptions occur after the fused image breaks up, around $18 \mathrm{msec}$.

The effect of intensity, on those types of judgments, is to alter the initial point at which various perceptions begin. For example, with decreases in intensity, the onset delay necessary for the fused image to appear as two stimuli and for either simultaneity or successiveness to begin increases.

Assuming that changes in response time reflect changes that are occurring within, as well as between, stages, these results suggest two conclusions:

(1) The differential effects of stimulus intensity and the onset delays on the latency of temporal order and fusion-nonfusion judgments suggest that the processes involved in these types of judgments are not the same, since the same variables resulted in different response time functions. While the onset delay variable had a significant effect on the fusionnonfusion task and had no such effect on judgments of temporal order, the influence on the processes that account for those decisions could be different. It could also be speculated that the processes that account for those decisions could be different. Additionally, for onset delays greater than $8 \mathrm{msec}$, the relationship between stimulus intensity and response time for the fusion task is similar to the inverse relationship between stimulus intensity and 
simple reaction time. However, that inverse relation does not occur for onset delays shorter than $8 \mathrm{msec}$. Consequently, the simple reaction time models, presently available, do not appear to be able to account for this finding.

(2) The major conclusion that can be drawn from the differential effects of the stimulus variables on the latency of order judgments and of fusion-nonfusion judgments is that these two tasks, as defined, do not appear to be sequential. However, at short onset delays and for onset delays longer than $28 \mathrm{msec}$, some type of sequential processing appears to be occurring. But there is also support for nonsequential processing for those onset delays that occur between 8 and $28 \mathrm{msec}$. Consequently, these results suggest some type of parallel processing, or a switching process, that is a function of the onset delay and intensity of the stimulus pair. Therefore, the hypothesis proposed by Sternberg and Knoll (1973) and Babkoff (1975) must be modified.

For short onset delays, lateralization and fusion are sequential, with the latter occurring second. As the onset delay increases, since temporal order judgments and nonfusion judgments require the same amount of processing time, either they occur during the same stage (which would suggest that they are the same type of decision) or they occur in different, parallel stages. Lastly, as the onset delay increases even more, nonfusion judgments and temporal order judgments are sequential, with the latter occurring second. These findings suggest some type of hybrid, partially sequential and partially parallel, model.

Another explanation for this date could perhaps be arrived at by simply postulating two parallel independent systems, one responsible for order of occurrence and the other responsible for the number of events that occur. However, somewhere in the processing sequence, both types of information would have to be integrated. While the exact nature of this processing is unknown, it does appear that order information and fusion-nonfusion information are to some extent dependent on each other. The extent of this dependency varies to a large degree on the interval separating the onset of the stimuli. Further in- vestigations being conducted in this laboratory may shed some light on the exact nature of the processing within the stages that deal with order and fusionnonfusion information.

\section{REFERENCES}

BaвкоғF, H. Diotic temporal interactions: Fusion and temporal order. Perception \& Psychophysics, 1975, 18, 267-272.

BaвкоFғ, H., \& Sutton, S. End points of lateralization for dichotic clicks. Journal of the Acoustical Society of America, 1966, 39, 87-102.

Cheatham, P., \& White, C. Temporal numerosity: III. Auditory perception of number. Journal of Experimental Psychology, $1954,47,425-428$.

Corso, G. M. Simultaneity, temporal order and stimulus intensity. Unpublished Master's thesis, New Mexico State University, 1976.

Corso, G. M. Psychological time. Unpublished doctoral dissertation, New Mexico State University, 1978.

Fraisse, P. The psychology of time. New York: Harper and Row, 1963.

Harris, J. D. Psychoacoustics. New York: Bobbs-Merrill, 1974.

Hinsh, I. Auditory perception of temporal order. Journal of the Acoustical Society of America, 1959, 31, 759-767.

Hirsh, I., \& Sherrick, C. Perceived order in different sense modalities. Journal of Experimental Psychology, 1961, 62 , 423-432.

Rutschmann, R. Visual perception of temporal order. In $\mathrm{S}$. Kornblum (Ed.), Attention and performance $I V$. New York: Academic Press, 1973.

SternberG, S., \& KNOLL, R. The perception of temporal order: Fundamental issues and a general model. In S. Kornblum (Ed.), Attention and performance IV. New York: Academic Press, 1973.

Stroud, J. The fine structure of psychological time. In $\mathrm{H}$. Quastler (Ed.), Information theory in psychology. Glenco, 1ll: Free Press, 1955.

Teichner, W., \& Kress, M. Laws of the simple visual reaction time. Psychological Review, 1972, 79, 344-358.

Wallach, H., Newman, E. B., \& Rosenzweig, M. R. Precedence effect in sound localization. American Journal of Psychology, 1949, 62, 315-336.

Wh ITE, C. T. Temporal numerosity and the psychological unit of duration. Psychological Monographs: General \& Applied, 1963, 77(12, Whole No. 575), 1-37.

White, C. T., \& Lichtenstein, M. Some aspects of temporal discrimination. Perceptual and Motor Skills, 1963, 17, 471-482.

(Received for publication December 7, 1979; revision accepted August 28, 1980.) 